\title{
Central Field in Rotating Spherical Space in 5D
}

\author{
Wladimir Be layev
}

Center for Relativity and Astrophysics, 185 Box, 194358, Saint-Petersburg, Russia

\begin{abstract}
A time-like geodesic motion in rotating 5D space is studied in framework of Kaluza-Klein theory and astrophysical applications are proposed. For example of such space-time in 4D spherical coordinates with the space-like fifth dimension a transition to the cylindrical frame is implemented. After this transformation a space is divided into two parts that is interpreted physically as pair of the universe and anti-universe. The rotation in 5D space-time exhibits itself in $4 \mathrm{D}$ as action of extra force, which reveals itself with observed radial motion of the material particle. A lso it is considered similar $(3+2) \mathrm{D}$ space-time in which additional dimension is time-like and a motion of space is hyperbolic. For these space-times it is found geodesics with constant radius in $4 \mathrm{D}$ spherical coordinates and studied their small deviations in cylindrical frames. It is shown that model with space-like fifth coordinate conforms to the basic properties of the Pioneer-effect, namely, a) constant additional acceleration of apparatus on distance from 20 to 50 a.e., b) its increase from 5 to 20 a.e., c) observed absence of one in motion of planets.
\end{abstract}

Keywords Time-Like Geodesics, Kalu za-Klein Theory, Pioneer-Effect

\section{Introduction}

A five-dimensional model of the space-time was proposed by Nordstrom[1] and Kaluza[2] for unity of gravitation and electromagnetism. Klein[3] suggested a compactification mechanis $\mathrm{m}$, owing to which internal space of the Planck size forms additional dimension. In his theory a motion of particle having rest mass in $4 \mathrm{D}$ can be described by equations of null geodesic line in 5D, which are interpretation of massless wave equation with some conditions.

In development of this model 5D space-time is considered as low energy limit of more high-dimensional theories of supersymmetry, supergravity and string theory. They admit scenario, in which particle has a rest mass in 5D[4, 5]. Exact solutions of Kaluza-Klein and low limit of bosonic string theories in $5 \mathrm{D}-6 \mathrm{D}[6]$ with to roidal compactification are equivalent. Analogous conclusion is made in[7] with comparison space-time-mass theory based on geometric properties of 5D space without compactification and braneworld model. Predictions of five-dimensional model of extended space and its experimental tests are considered in $[8$, 9]. Cos mological model with motion of matter in fifth dimension also is examined[10]. Astrophysical applications of braneworld theories, including Arkani-HamedDimopoulos-Dvali and Einstein-Maxwell models with large extra dimensions, are analyzed in[11]. EM model in $6 \mathrm{D}$ has become further development in[12], where linear perturbations sourced by matter on the brane are studied.

* Corresponding author:

wbelayev@yandex.ru (Wladimir Belayev)

Published online at http://journal.sapub.org/ijtmp

Copyright (C) 2012 Scientific \& Academic Publishing. All Rights Reserved
$\operatorname{In}[13]$ it is proposed low energy effective theory on a regularized brane in $6 \mathrm{D}$ gauged chiral supergravity. A possibility of the orbits of particles around the extra dimesions in $(4+n) D$ space, periodically returning in 4D surface,

is phenomenologically predicted in ADD model[14]. Phenomena, described by one-time physics in $3+1$ dimensions, appear as various "shadows" similar phenomena that occur in $4+2$ dimensions with one extra space and one extra time dimensions (more generally, $\mathrm{d}+2)[15,16,17]$.

In present paper it is considered some geometrical construction in $(4+1) \mathrm{D}$ space-time with space-like fifth dimension and rotation in 4D spherical coordinates with transition thereupon to the standard cylindrical frame. It is studied also $(3+2) \mathrm{D}$ space-time with time-like additional dimension, where the motion is hyperbolic.

A motion of the particle in certain domain of space in appropriate coordinates is assigned to be described with sufficient accuracy by geodesic equations. Their solutions lead to conclusion that rotation in $5 \mathrm{D}$ space-time exhibits itself in 4D as action of central force.

In astrophysical applications proposed model of space-time is of interest with respect to Pioneer effect. In some papers[18, 19], see also review of efforts to explain anomaly[20], presence of signal frequency bias is associated with dependence of fundamental physical parameters from time. Though it should allow for data[21], which witness independence of direction of additional acceleration from route of radial motion with respect to Sun. A radial Rindler-like acceleration[22, 23] in itself also can't expla in the Pioneer effect because it is absent in planets motion[24].

\section{Geodesics in Rotating Space}


Five-dimensional space-time having 4D spherical symmetry is considered in coordinate frame $X_{s}^{i}=(\tau, a, \theta, \varphi, \chi)$, where $a, \theta, \varphi, \chi$ are spherical space coordinates and $\tau=c t$, where $c$ is light velocity constant and $t$ is time. Rotating space-time with space-like fifth dimension[25] is described by metric

$$
\begin{gathered}
d S^{2}=\left[1-a^{2} B(a)^{2}\right] d \tau^{2}-d a^{2}-a^{2}[2 B(a) g(\chi) d \tau d \chi+ \\
\left.+\sin ^{2} f(\chi)\left(d \theta^{2}+\sin ^{2} \theta d \varphi^{2}\right)+g^{2}(\chi) d \chi^{2}\right]
\end{gathered}
$$

where function $f(\chi)$ is continuously increasing and it is taken $g=d f / d \chi$. For the domain under review it is assumed

$$
B(a)=K a^{-1 / 2},
$$

where $K$ is constant.

Transition to five-dimensional cylindrical coordinates $X_{c}^{i}=(\tau, r, \theta, \varphi, y)$ for $0 \leq \chi \leq \pi$ is performed by transformation

$$
r=a \sin f(\chi), y=a \cos f(\chi) .
$$

Geodesic equations in 5D for particle having rest mass are

$$
\frac{d U^{i}}{d S}+\Gamma_{k l}^{i} U^{k} U^{l}=0
$$

where $U^{i}$ are components of five-velocity vector and $\Gamma_{k l}^{i}$ are 5D Christoffel symbols of second kind. In spherical coordinates for metric (1) these equations, with

$$
f(\chi)=\chi \text {, }
$$

take form

$$
\begin{gathered}
\frac{d^{2} \tau}{d S^{2}}+\frac{K^{2}}{2} \frac{d \tau}{d S} \frac{d a}{d S}+\frac{K a^{1 / 2}}{2} \frac{d a}{d S} \frac{d \chi}{d S}- \\
-\frac{K a^{3 / 2}}{2} \sin (2 \chi)\left(\frac{d \theta}{d S}\right)^{2}-\frac{K a^{3 / 2}}{2} \sin (2 \chi) \sin ^{2} \varphi\left(\frac{d \varphi}{d S}\right)^{2}=0,(6) \\
-a \sin ^{2} \chi\left(\frac{d \theta}{d S}\right)^{2}-a \sin ^{2} \theta \sin ^{2} \chi\left(\frac{d \varphi}{d S}\right)^{2}-a\left(\frac{d \chi}{d S}\right)^{2}=0, \\
\frac{d^{2} \theta}{d S^{2}}+\frac{2}{a} \frac{d a}{d S} \frac{K^{2}}{d S}\left(\frac{d \tau}{d S}\right)^{2}-\frac{3 K a^{1 / 2}}{2} \frac{d \tau}{d S} \frac{d \chi}{d S}- \\
-\frac{d^{2} \varphi}{d S^{2}}+\frac{2}{a} \frac{d a}{d S} \frac{d \varphi}{d S}+2 \cot \theta \frac{d \theta}{d S} \frac{d \varphi}{d S}+2 \cot \chi \frac{d \varphi}{d S} \frac{d \chi}{d S}=0 \\
\frac{d^{2} \chi}{d S^{2}}+\frac{K(3)}{d S}-\frac{\sin (2 \theta)}{2}\left(\frac{d \varphi}{d S}\right)^{2}=0 \\
\left.2 a^{3 / 2} a\right)
\end{gathered}
$$

For particle with a rest mass the solutions of these equations must correspond to given by metric condition

$$
\begin{gathered}
1=\left(1-K^{2} a\right) U^{02}-2 K a^{3 / 2} U^{0} U^{4}-U^{12}- \\
-\sin ^{2} \chi a^{2}\left(U^{22}+\sin ^{2} \theta U^{32}\right)-a^{2} U^{42} .
\end{gathered}
$$

Fro $m$ the second equation of the system we obtain

$$
\begin{aligned}
U^{4}= & \frac{K U^{0}}{4 a^{1 / 2}}\left\{-3+\mu\left[1+\frac{16}{a K^{2} U^{02}}\left(\frac{d U^{1}}{d S}-\right.\right.\right. \\
& \left.\left.\left.-a \sin ^{2} \chi\left(U^{22}+\sin ^{2} \theta U^{32}\right)\right)\right]^{1 / 2}\right\}
\end{aligned}
$$

where $\mu$ is \pm 1 . We name corresponding solutions as a solution of type I with $\mu=-1$ and as a solution of type II with $\mu=1$.

When particles move along geodesics, which are arcs of circle:

$$
U^{1}=U^{2}=U^{3}=0,
$$

equations of motion have following solutions:

$$
U_{I}^{0}=\sigma, U_{I}^{4}=-\frac{\sigma K}{a^{1 / 2}}
$$

and

$$
U_{I I}^{0}=\frac{2 \sigma}{\sqrt{4-K^{2} a}}, U_{I I}^{4}=-\frac{\sigma K}{a^{1 / 2} \sqrt{4-K^{2} a}},
$$

where $\sigma$ is $1,-1$.

Change of passage of time is defined as relation between intervals of proper time $T=\int d S$ and coordinate time $\tau$.

For geodesic of type I for solution (14) chosen $\sigma=1$ we have

$$
d \tau=d T
$$

i.e. time dilation is absence. With motion of particle along circular geodesic of type II (15) we obtain

$$
d T=\frac{1}{2} \sqrt{4-K^{2} a} d \tau \text {. }
$$

\section{Representation in Cylindrical Frame}

After substitutions of coordinate transformation being inverse to (3), namely,

$$
a=\sqrt{r^{2}+y^{2}}, f(\chi)=\operatorname{arccot} \frac{y}{r}
$$

metric (1) with (2) is rewritten as

$$
\begin{aligned}
d S^{2} & =\left(1-K^{2} \sqrt{r^{2}+y^{2}}\right) d \tau^{2}-2 K\left(r^{2}+y^{2}\right)^{-1 / 4} d \tau(y d r- \\
& -r d y)-d r^{2}-r^{2}\left(d \theta^{2}+\sin ^{2} \theta d \varphi^{2}\right)-d y^{2}
\end{aligned}
$$

Geodesic equations will be

$$
\frac{d^{2} \tau}{d S^{2}}+\frac{K^{2} r}{2 \sqrt{r^{2}+y^{2}}} \frac{d \tau}{d S} \frac{d r}{d S}+\frac{K^{2} y}{2 \sqrt{r^{2}+y^{2}}} \frac{d \tau}{d S} \frac{d y}{d S}+
$$




$$
\begin{gathered}
+\frac{K r y}{2\left(r^{2}+y^{2}\right)^{5 / 4}}\left(\frac{d r}{d S}\right)^{2}-\frac{K\left(r^{2}-y^{2}\right)}{2\left(r^{2}+y^{2}\right)^{5 / 4}} \frac{d r}{d S} \frac{d y}{d S}- \\
-\frac{K r y}{\left(r^{2}+y^{2}\right)^{1 / 4}}\left(\frac{d \theta}{d S}\right)^{2}-\frac{K r y}{\left(r^{2}+y^{2}\right)^{1 / 4} \sin ^{2} \varphi\left(\frac{d \varphi}{d S}\right)^{2}-} \\
-\frac{K r y}{2\left(r^{2}+y^{2}\right)^{5 / 4}}\left(\frac{d y}{d S}\right)^{2}=0 \\
\frac{d^{2} r}{d S^{2}}-\frac{K^{2} r}{2 \sqrt{r^{2}+y^{2}}}\left(\frac{d \tau}{d S}\right)^{2}-\frac{K^{3} r y}{2\left(r^{2}+y^{2}\right)^{3 / 4}} \frac{d \tau}{d S} \frac{d r}{d S}- \\
-\frac{K^{3} y^{2}-3 K \sqrt{r^{2}+y^{2}}}{2\left(r^{2}+y^{2}\right)^{3 / 4}} \frac{d \tau}{d S} \frac{d y}{d S}-\frac{K^{2} r y^{2}}{2\left(r^{2}+y^{2}\right)^{3 / 2}}\left(\frac{d r}{d S}\right)^{2}+ \\
+\frac{K^{2} y\left(r^{2}-y^{2}\right)}{2\left(r^{2}+y^{2}\right)^{3 / 2}} \frac{d r}{d S} \frac{d y}{d S}+\frac{r\left(K^{2} y^{2}-\sqrt{r^{2}+y^{2}}\right)}{\sqrt{r^{2}+y^{2}}}\left(\frac{d \theta}{d S}\right)^{2}+ \\
+\frac{r\left(K^{2} y^{2}-\sqrt{r^{2}+y^{2}}\right)}{\sqrt{r^{2}+y^{2}}} \sin ^{2} \theta\left(\frac{d \varphi}{d S}\right)^{2}+\frac{K^{2} r y^{2}}{2\left(r^{2}+y^{2}\right)^{3 / 2}}\left(\frac{d y}{d S}\right)^{2}=0,(21) \\
+\frac{K^{3} r y}{2\left(r^{2}+y^{2}\right)^{3 / 4}} \frac{d \tau}{d S} \frac{d y}{d S}+\frac{K^{2} r^{2} y}{2\left(r^{2}+y^{2}\right)^{3 / 2}}\left(\frac{d \theta}{\sqrt{r^{2}+y^{2}}}\right)^{2}-\frac{K^{2} r^{2} y}{\sqrt{r^{2}+y^{2}}} \sin ^{2} \theta\left(\frac{d \varphi}{d S}\right)^{2}-\frac{K^{2} K^{2} r\left(r^{2}-y^{2}\right)}{2\left(r^{2}+y^{2}\right)^{3 / 2}}\left(\frac{d y}{d S}\right)^{2}=0 \\
\frac{d^{2} y}{d S^{2}}-\frac{d r}{r} \frac{K^{2} y}{d S} \frac{d y}{d S}-\frac{\sin ^{2}(2 \theta)}{2}\left(\frac{d \varphi}{d S}\right)^{2}=0 \\
\frac{d^{2} \varphi}{d S^{2}}+\frac{2}{r} \frac{d r}{d S} \frac{d \varphi}{d S}+2 \cot \theta \frac{d \theta}{d S} \frac{d \varphi}{d S}=0,
\end{gathered}
$$

Components of five-velocity vector corresponding to coordinates $r$ and $y$ are found by differentiation of transformations (3) and will be

$$
V^{1}=\sin \chi U^{1}+a \cos \chi U^{4}, V^{4}=\cos \chi U^{1}-a \sin \chi U^{4} .
$$

Condition given by metric (19) for the time-like path is

$$
\begin{aligned}
1= & \left(1-K^{2} \sqrt{r^{2}+y^{2}}\right) V^{02}-2 K\left(r^{2}+y^{2}\right)^{-1 / 4} V^{0}\left(y V^{1}-\right. \\
& \left.-r V^{4}\right)-V^{12}-r^{2}\left(V^{22}+\sin ^{2} \theta V^{32}\right)-V^{42} .
\end{aligned}
$$

The non-zero components of five-velocity vector corresponding to solutions of geodesic equations in coordinates $X_{s}$ (13)-(15) are rewritten in coordinate frame $X_{c}$ as

$$
V_{I}^{0}=\sigma, V_{I}^{1}=-\frac{\sigma K y}{\left(r^{2}+y^{2}\right)^{1 / 4}}, V_{I}^{4}=\frac{\sigma K r}{\left(r^{2}+y^{2}\right)^{1 / 4}}
$$

$$
\begin{gathered}
V_{I I}^{0}=\frac{2 \sigma}{\left(4-K^{2} \sqrt{r^{2}+y^{2}}\right)^{1 / 2}}, \\
V_{I I}^{1}=-\frac{\sigma K y}{\left(r^{2}+y^{2}\right)^{1 / 4}\left(4-K^{2} \sqrt{r^{2}+y^{2}}\right)^{1 / 2}}, \\
V_{I I}^{4}=\frac{\sigma K r}{\left(r^{2}+y^{2}\right)^{1 / 4}\left(4-K^{2} \sqrt{r^{2}+y^{2}}\right)^{1 / 2}} .
\end{gathered}
$$

For $y=0 \quad(\chi=\pi / 2)$ they correspond with stationary in 4D particle.

For motion in the neighborhood of point $\left(\tau_{0}, r_{0}, \pi / 2,0,0\right)$ with $V_{0}^{2}=V_{0}^{3}=0$ geodesic equations are reduced to

$$
\begin{gathered}
\frac{d^{2} \tau}{d S^{2}}+\frac{K^{2}}{2} \frac{d \tau}{d S} \frac{d r}{d S}-\frac{K}{2 r_{0}^{1 / 2}} \frac{d r}{d S} \frac{d y}{d S}=0, \\
\frac{d^{2} r}{d S^{2}}-\frac{K^{2}}{2}\left(\frac{d \tau}{d S}\right)^{2}+\frac{3 K}{2 r_{0}^{1 / 2}} \frac{d \tau}{d S} \frac{d y}{d S}=0, \\
\frac{d^{2} y}{d S^{2}}+\frac{K^{3} r_{0}-3 K}{2 r_{0}^{1 / 2}} \frac{d \tau}{d S} \frac{d r}{d S}-\frac{K^{2}}{2} \frac{d r}{d S} \frac{d y}{d S}=0 .
\end{gathered}
$$

Condition (26) takes form

$$
1=\left(1-K^{2} r_{0}\right) V^{02}-2 K r_{0}^{1 / 2} V^{0} V^{4}-V^{12}-V^{42} \text {. }
$$

For solutions of type I and II (27)-(30) be ing circular motion Eq. (21) y ields non-vanishing radial accele rations

$$
\begin{gathered}
\frac{d V_{I}^{1}}{d S}=-K^{2}, \\
\frac{d V_{I I}^{1}}{d S}=-\frac{K^{2}}{4-K^{2} \sqrt{r^{2}+y^{2}}} .
\end{gathered}
$$

\section{Metrics with Time-Like Fifth Coordinate}

A space-time with 4D spherical coordinates $\breve{X}_{g}^{i}=(\breve{\tau}, \breve{a}, \breve{\theta}, \breve{\varphi}, \breve{\chi})$ having hyperbolic motion is described by metric

$$
\begin{aligned}
d S^{2}= & \left(1+\breve{K}^{2} \breve{a}\right) d \breve{\tau}^{2}-d \breve{a}^{2}+\breve{a}^{2}\left[2 \breve{K} \breve{a}^{-1 / 2} d \breve{\tau} d \breve{\chi}-\right. \\
& \left.-\cosh \breve{\chi}^{2}\left(d \breve{\theta}^{2}+\sin ^{2} \breve{\theta} d \breve{\varphi}^{2}\right)+d \breve{\chi}^{2}\right],
\end{aligned}
$$

where $\breve{\chi}$ is assumed to be time-like and $\breve{K}$ is constant. This metric can be obtained from (1) for (2), (5) by substitution $K=-\mathrm{i} \breve{K}, \chi=\frac{\pi}{2}-\mathrm{i} \breve{\chi}$ and addition of ( $)$ in the notation of other coordinates.

The geodesics equations for a particle motion along time-like path are

$\frac{d^{2} \breve{\tau}}{d S^{2}}-\frac{\breve{K}^{2}}{2} \frac{d \breve{\tau}}{d S} \frac{d \breve{a}}{d S}-\frac{\breve{K} \breve{a}^{1 / 2}}{2} \frac{d \breve{a}}{d S} \frac{d \breve{\chi}}{d S}-\frac{\breve{K} \breve{a}^{3 / 2}}{2} \sinh (2 \breve{\chi})\left(\frac{d \breve{\theta}}{d S}\right)^{2}-$ 


$$
\begin{gathered}
-\frac{\breve{K} \breve{a}^{3 / 2}}{2} \sinh (2 \breve{\chi}) \sin ^{2} \breve{\varphi}\left(\frac{d \breve{\varphi}}{d S}\right)^{2}=0, \\
\frac{d^{2} \breve{a}}{d S^{2}}+\frac{\breve{K}^{2}}{2}\left(\frac{d \breve{\tau}}{d S}\right)^{2}+\frac{3 \breve{K} \breve{a}^{1 / 2}}{2} \frac{d \breve{\tau}}{d S} \frac{d \breve{\chi}}{d S}-\breve{a} \cosh ^{2} \breve{\chi}\left(\frac{d \breve{\theta}}{d S}\right)^{2}- \\
-\breve{a} \sin ^{2} \breve{\theta} \cosh ^{2} \breve{\chi}\left(\frac{d \breve{\varphi}}{d S}\right)^{2}+\breve{a}\left(\frac{d \breve{\chi}}{d S}\right)^{2}=0, \\
\frac{d^{2} \breve{\theta}}{d S^{2}}+\frac{2}{\breve{a}} \frac{d \breve{a}}{d S} \frac{d \breve{\theta}}{d S}+2 \tanh \breve{\chi} \frac{d \breve{\theta}}{d S} \frac{d \breve{\chi}}{d S}-\frac{\sin (2 \breve{\theta})}{2}\left(\frac{d \breve{\varphi}}{d S}\right)^{2}=0, \\
\frac{d^{2} \breve{\varphi}}{d S^{2}}+\frac{2}{\breve{a}} \frac{d \breve{a}}{d S} \frac{d \breve{\varphi}}{d S}+2 \cot \breve{\theta} \frac{d \breve{\theta}}{d S} \frac{d \breve{\chi}}{d S}+2 \tanh \breve{\chi} \frac{d \breve{\varphi}}{d S} \frac{d \breve{\chi}}{d S}=0, \\
\frac{d^{2} \breve{\chi}}{d S^{2}}+\frac{\breve{K}\left(3+\breve{K}^{2} \breve{a}\right)}{2 \breve{a}^{3 / 2}} \frac{d \breve{\tau}}{d S} \frac{d \breve{a}}{d S}+\frac{4+\breve{K} \breve{a}^{2} \breve{a} \frac{d \breve{a}}{d S} \frac{d \breve{\chi}}{d S}+}{2} \\
+\frac{\left(1+\breve{K}^{2} \breve{a}\right) \sinh (2 \breve{\chi})}{2}\left(\frac{d \breve{\theta}}{d S}\right)^{2}+ \\
\frac{\left(1+\breve{K}^{2} \breve{a}\right) \sin ^{2} \breve{\theta} \sinh (2 \breve{\chi})}{2}\left(\frac{d \breve{\varphi}}{d S}\right)^{2}=0 .
\end{gathered}
$$

For particle with the rest mass the solutions of these equations must correspond to given by metric condition

$$
\begin{gathered}
1=\left(1+\breve{K}^{2} \breve{a}\right) \breve{U}^{02}+2 \breve{K} \breve{a}^{3 / 2} \breve{U}^{0} \breve{U}^{4}-\breve{U}^{12}- \\
-\cosh ^{2} \breve{\chi} \breve{a}^{2}\left(\breve{U}^{22}+\sin ^{2} \breve{\theta} \breve{U}^{32}\right)+\breve{a}^{2} \breve{U}^{42} .
\end{gathered}
$$

Second equation of the system yields

$$
\begin{aligned}
\breve{U}^{4} & =\frac{\breve{K} \breve{U}^{0}}{4 \breve{a}^{1 / 2}}\left\{-3+\mu\left[1-\frac{16}{\breve{a} \breve{K}^{2} \breve{U}^{02}}\left(\frac{d \breve{U}^{1}}{d S}-\right.\right.\right. \\
& \left.\left.\left.-\breve{a} \cosh ^{2} \breve{\chi}\left(\breve{U}^{22}+\sin ^{2} \breve{\theta} \breve{U}^{32}\right)\right)\right]^{1 / 2}\right\}
\end{aligned}
$$

With hyperbolic motion for $\breve{U}^{1}=\breve{U}^{2}=\breve{U}^{3}=0$ corresponding five-velocity vectors have non-zero components

$$
\begin{gathered}
\breve{U}_{I}^{0}=\sigma, \breve{U}_{I}^{4}=-\frac{\sigma \breve{K}}{\breve{a}^{1 / 2}}, \\
\breve{U}_{I I}^{0}=\frac{2 \sigma}{\sqrt{4+\breve{K}^{2} \breve{a}}}, \breve{U}_{I I}^{4}=-\frac{\sigma \breve{K}}{\breve{a}^{1 / 2} \sqrt{4+\breve{K}^{2} \breve{a}}} .
\end{gathered}
$$

For solution of type I time dilation is absent:

$$
d \breve{T}=d \breve{\tau},
$$

and for solution of type II the increase of passage of the proper time is given by

$$
d \breve{T}=\frac{1}{2} \sqrt{4+\breve{K}^{2} \breve{a}} d \breve{\tau} .
$$

Transition to cylindrical coordinates $\breve{X}_{c}^{i}=(\breve{\tau}, \breve{r}, \breve{\theta}, \breve{\varphi}, \breve{y})$ is realized by transformation $\breve{r}=\breve{a} \cosh \breve{\chi}, \breve{y}=\breve{a} \sinh \breve{\chi}$.
Corresponding components of the five-velocity vector are $\breve{V}^{1}=\cosh \breve{\chi} \breve{U}^{1}+\breve{a} \sinh \breve{\chi} \breve{U}^{4}, \breve{V}^{4}=\sinh \breve{\chi} \breve{U}^{1}+\breve{a} \cosh \breve{\chi} \breve{U}^{4}$. (50)

Inverse coordinate transformation is written as

$$
\breve{a}=\sqrt{\breve{r}^{2}-\breve{y}^{2}}, \breve{\chi}=\operatorname{arcoth} \frac{\breve{y}}{\breve{r}} .
$$

Substituting this in (37) gives

$$
\begin{aligned}
d S^{2} & =\left(1+\breve{K}^{2} \sqrt{\breve{r}^{2}-\breve{y}^{2}}\right) d \breve{\tau}^{2}-2 \breve{K}\left(\breve{r}^{2}-\breve{y}^{2}\right)^{-1 / 4} d \breve{\tau}(\breve{y} d \breve{r}- \\
& -\breve{r} d \breve{y})-d \breve{r}^{2}-\breve{r}^{2}\left(d \breve{\theta}^{2}+\sin ^{2} \breve{\theta} d \breve{\varphi}^{2}\right)+d \breve{y}^{2} .
\end{aligned}
$$

The same line element can be obtained by replacement $K=-\mathrm{i} \breve{K}, y=\mathrm{i} \breve{y}$ and addition of ( $)$ in the notation of other coordinates in (19).

Geodesics equations for motion of particle having rest mass are written as

$$
\begin{gathered}
\frac{d^{2} \breve{\tau}}{d S^{2}}-\frac{\breve{K}^{2} \breve{r}}{2 \sqrt{\breve{r}^{2}-\breve{y}^{2}}} \frac{d \breve{\tau}}{d S} \frac{d \breve{r}}{d S}+\frac{\breve{K}^{2} \breve{y}}{2 \sqrt{\breve{r}^{2}-\breve{y}^{2}}} \frac{d \breve{\tau}}{d S} \frac{d \breve{y}}{d S}+ \\
+\frac{\breve{K} \breve{y}}{2\left(\breve{r}^{2}-\breve{y}^{2}\right)^{5 / 4}}\left(\frac{d \breve{r}}{d S}\right)^{2}-\frac{\breve{K}\left(\breve{r}^{2}+\breve{y}^{2}\right)}{2\left(\breve{r}^{2}-\breve{y}^{2}\right)^{5 / 4}} \frac{d \breve{r}}{d S} \frac{d \breve{y}}{d S}- \\
-\frac{\breve{K} \breve{y}}{c\left(\breve{r}^{2}-\breve{y}^{2}\right)^{1 / 4}}\left(\frac{d \breve{\theta}}{d S}\right)^{2}-\frac{\breve{K} \breve{y}}{\left(\breve{r}^{2}-\breve{y}^{2}\right)^{1 / 4}} \sin ^{2} \breve{\varphi}\left(\frac{d \breve{\varphi}}{d S}\right)^{2}+ \\
+\frac{\breve{K} \breve{r} \breve{y}}{2\left(\breve{r}^{2}-\breve{y}^{2}\right)^{5 / 4}}\left(\frac{d \breve{y}}{d S}\right)^{2}=0,
\end{gathered}
$$

$\frac{d^{2} \breve{r}}{d S^{2}}+\frac{\breve{K}^{2} \breve{r}}{2 \sqrt{\breve{r}^{2}-\breve{y}^{2}}}\left(\frac{d \breve{\tau}}{d S}\right)^{2}+\frac{\breve{K}^{3} \breve{r} \breve{y}}{2\left(\breve{r}^{2}-\breve{y}^{2}\right)^{3 / 4}} \frac{d \breve{\tau}}{d S} \frac{d \breve{r}}{d S}-$ $-\frac{\breve{K}^{3} \breve{y}^{2}-3 \breve{K} \sqrt{\breve{r}^{2}-\breve{y}^{2}}}{2\left(\breve{r}^{2}-\breve{y}^{2}\right)^{3 / 4}} \frac{d \breve{\tau}}{d S} \frac{d \breve{y}}{d S}-\frac{\breve{K}^{2} \breve{r}^{2}}{2\left(\breve{r}^{2}-\breve{y}^{2}\right)^{3 / 2}}\left(\frac{d \breve{r}}{d S}\right)^{2}+$ $+\frac{\breve{K}^{2} \breve{y}\left(\breve{r}^{2}+\breve{y}^{2}\right)}{2\left(\breve{r}^{2}-\breve{y}^{2}\right)^{3 / 2}} \frac{d \breve{r}}{d S} \frac{d \breve{y}}{d S}+\frac{\breve{r}\left(\breve{K}^{2} \breve{y}^{2}-\sqrt{\breve{r}^{2}-\breve{y}^{2}}\right)}{\sqrt{\breve{r}^{2}-\breve{y}^{2}}}\left(\frac{d \breve{\theta}}{d S}\right)^{2}+$ $+\frac{\breve{r}\left(\breve{K}^{2} \breve{y}^{2}-\sqrt{\breve{r}^{2}-\breve{y}^{2}}\right)}{\sqrt{\breve{r}^{2}-\breve{y}^{2}}} \sin ^{2} \breve{\theta}\left(\frac{d \breve{\varphi}}{d S}\right)^{2}-\frac{\breve{K}^{2} \breve{r} \breve{y}^{2}}{2\left(\breve{r}^{2}-\breve{y}^{2}\right)^{3 / 2}}\left(\frac{d \breve{y}}{d S}\right)^{2}=0$,

$$
\frac{d^{2} \breve{\theta}}{d S^{2}}+\frac{2}{\breve{r}} \frac{d \breve{r}}{d S} \frac{d \breve{\theta}}{d S}-\frac{\sin (2 \breve{\theta})}{2}\left(\frac{d \breve{\varphi}}{d S}\right)^{2}=0,
$$

$$
\frac{d^{2} \breve{\varphi}}{d S^{2}}+\frac{2}{\breve{r}} \frac{d \breve{r}}{d S} \frac{d \breve{\varphi}}{d S}+2 \cot \breve{\theta} \frac{d \breve{\theta}}{d S} \frac{d \breve{\varphi}}{d S}=0,
$$

$\frac{d^{2} \breve{y}}{d S^{2}}+\frac{\breve{K}^{2} \breve{y}}{2 \sqrt{\breve{r}^{2}-\breve{y}^{2}}}\left(\frac{d \breve{\tau}}{d S}\right)^{2}+\frac{\breve{K}^{3} \breve{r}^{2}+3 \breve{K} \sqrt{\breve{r}^{2}-\breve{y}^{2}}}{2\left(\breve{r}^{2}-\breve{y}^{2}\right)^{3 / 4}} \frac{d \breve{\tau}}{d S} \frac{d \breve{r}}{d S}-$ $-\frac{\breve{K}^{3} \breve{r} \breve{y}}{2\left(\breve{r}^{2}-\breve{y}^{2}\right)^{3 / 4}} \frac{d \widetilde{\tau}}{d S} \frac{d \bar{y}}{d S}-\frac{\breve{K}^{2} \breve{y r}^{2}}{2\left(\breve{r}^{2}-\breve{y}^{2}\right)^{3 / 2}}\left(\frac{d \breve{r}}{d S}\right)^{2}+\frac{\breve{K}^{2} \breve{r}\left(\breve{r}^{2}+\breve{y}^{2}\right)}{2\left(\breve{r}^{2}-\breve{y}^{2}\right)^{3 / 2}} \frac{d \breve{r}}{d S} \frac{d \breve{y}}{d S}+$ $+\frac{\breve{K}^{2} \breve{r}^{2} \breve{y}}{\sqrt{\breve{r}^{2}-\breve{y}^{2}}}\left(\frac{d \breve{\theta}}{d S}\right)^{2}+\frac{\breve{K}^{2} \breve{r}^{2} \breve{y}}{\sqrt{\breve{r}^{2}-\breve{y}^{2}}} \sin ^{2} \breve{\theta}\left(\frac{d \breve{\varphi}}{d S}\right)^{2}-\frac{\breve{K}^{2} \breve{r}^{2} \breve{y}}{2\left(\breve{r}^{2}-\breve{y}^{2}\right)^{3 / 2}}\left(\frac{d \breve{y}}{d S}\right)^{2}=0$. 
Condition given by metric (52) for the time-like path is $1=\left(1+\breve{K}^{2} \sqrt{\breve{r}^{2}-\breve{y}^{2}}\right) \breve{V}^{02}-2 \breve{K}\left(\breve{r}^{2}-\breve{y}^{2}\right)^{-1 / 4} \breve{V}^{0}\left(\breve{y} \breve{V}^{1}-\right.$

$$
\left.-\breve{r} \breve{V}^{4}\right)-\breve{V}^{12}-\breve{r}^{2}\left(\breve{V}^{22}+\sin ^{2} \breve{\theta} \breve{V}^{32}\right)+\breve{V}^{42} \text {. }
$$

A non-zero components of five-velocity vectors corresponding hyperbolic solutions (45), (46) are

$$
\begin{gathered}
\breve{V}_{I}^{0}=\sigma, \breve{V}_{I}^{1}=-\frac{\sigma \breve{K} \widetilde{y}}{\left(\breve{r}^{2}-\breve{y}^{2}\right)^{1 / 4}}, \breve{V}_{I}^{4}=-\frac{\sigma \breve{K} r}{\left(\breve{r}^{2}-\breve{y}^{2}\right)^{1 / 4}}, \\
\breve{V}_{I I}^{0}=\frac{2 \sigma}{\left(4+\breve{K}^{2} \sqrt{\breve{r}^{2}-\breve{y}^{2}}\right)^{1 / 2}}, \\
\breve{V}_{I I}^{1}=-\frac{\sigma \breve{K} \bar{y}}{\left(\breve{r}^{2}-\breve{y}^{2}\right)^{1 / 4}\left(4+\breve{K}^{2} \sqrt{\breve{r}^{2}-\breve{y}^{2}}\right)^{1 / 2}}, \\
\breve{V}_{I I}^{4}=-\frac{\sigma \breve{K}}{\left(\breve{r}^{2}-\breve{y}^{2}\right)^{1 / 4}\left(4+\breve{K}^{2} \sqrt{\left.\breve{r}^{2}-\breve{y}^{2}\right)^{1 / 2}}\right.} .
\end{gathered}
$$

For motion in the neighborhood of point $\left(\breve{\tau}_{0}, \breve{r}_{0}, \pi / 2,0,0\right)$ with $\breve{V}_{0}^{2}=\breve{V}_{0}^{3}=0$ local solution is found from reduced Eqs. (53)-(57), which turned out to

$$
\begin{gathered}
\frac{d^{2} \breve{\tau}}{d S^{2}}-\frac{\breve{K}^{2}}{2} \frac{d \breve{\tau}}{d S} \frac{d \breve{r}}{d S}-\frac{\breve{K}}{2 \breve{r}_{0}^{1 / 2}} \frac{d \breve{r}}{d S} \frac{d \breve{y}}{d S}=0, \\
\frac{d^{2} \breve{r}}{d S^{2}}+\frac{\breve{K}^{2}}{2}\left(\frac{d \breve{\tau}}{d S}\right)^{2}+\frac{3 \breve{K}}{2 \breve{r}_{0}^{1 / 2}} \frac{d \breve{\tau}}{d S} \frac{d \breve{y}}{d S}=0, \\
\frac{d^{2} \breve{y}}{d S^{2}}+\frac{\breve{K}^{3} \breve{r}_{0}+3 \breve{K}}{2 \breve{r}_{0}^{1 / 2}} \frac{d \breve{\tau}}{d S} \frac{d \breve{r}}{d S}+\frac{\breve{K}^{2}}{2} \frac{d \breve{r}}{d S} \frac{d \breve{y}}{d S}=0 .
\end{gathered}
$$

Condition (58) takes form

$$
1=\left(1+\breve{K}^{2} \breve{r}_{0}\right) \breve{V}^{02}+2 \breve{K}_{0}^{1 / 2} \breve{V}^{0} \breve{V}^{4}-\breve{V}^{12}+\breve{V}^{42} \text {. }
$$

For solutions of type I and II Eqs. (59)-(62) non-vanishing radial accelerations are

$$
\frac{d \breve{V}_{I}^{1}}{d S}=\breve{K}^{2}, \quad \frac{d \breve{V}_{I I}^{1}}{d S}=\frac{\breve{K}^{2}}{4+\breve{K}^{2} \sqrt{\breve{r}^{2}-\breve{y}^{2}}} .
$$

\section{Kaluza-Klein Model}

In Kaluza-Klein theory the line element is brought in form

$$
d^{2} S=g_{i j} d x^{i} d x^{j}+\varepsilon \Phi^{2}\left(A_{i} d x^{i}+\varepsilon d y\right)^{2},
$$

where $x^{i}$ and $g_{i j}$ are coordinates and metrical tensor of 4D space-time, $\Phi$ and $A$ are scalar and vector potential. Metrical coefficients and potentials are functions of $x^{i}$ and $y$. Constant $\varepsilon$ equals 1 for time-like fifth coordinate $y$ and -1 , when it is space-like.

In this form metrics (19) and (52) are represented by line-element of 4D space-time

$$
d s^{2}=\left(1-K^{2} \frac{y^{2}}{a_{\varepsilon}}\right) d \tau^{2}-2 K a_{\varepsilon}^{-1 / 2} y d \tau d r-d r^{2}-
$$

$$
-r^{2}\left(d \theta^{2}+\sin ^{2} \theta d \varphi^{2}\right)
$$

and potentials

$$
A_{0}=K r a_{\varepsilon}^{-1 / 2}, A_{1}=A_{2}=A_{3}=0, \Phi=1,
$$

where it is denoted $a_{\varepsilon}=\sqrt{r^{2}-\varepsilon y^{2}}$.

If $4 \mathrm{D}$ metric satisfies cylindrical conditions $\frac{\partial g_{i j}}{\partial y}=0$ ratio of electric charge to mass in $4 \mathrm{D}$ is written as

$$
\frac{q}{m}=\frac{Q}{\sqrt{1-Q^{2}}}
$$

with scalar function

$$
Q=\varepsilon \Phi^{2}\left(\frac{d y}{d S}+A_{i} \frac{d x^{i}}{d S}\right)
$$

In more general case with 4D metrical coefficients being dependent on $y$ the relationship between $Q$ and $q / m$ is not identical[4], but value $Q=0$ also corresponds to $q=0$.

For considering space-time after substituting components of the found five-velocity vectors of the type I (27) and (59) we obtain

$$
Q_{I}=0 \text {. }
$$

This value is interpreted as neutral charge of a test particle. For solutions of the type II (28)-(30) and (60)-(62) scalar function is

$$
Q_{I I}=\frac{\varepsilon \sigma K r}{a_{\varepsilon}^{1 / 2} \sqrt{4+\varepsilon K^{2} a_{\varepsilon}}} .
$$

The light trajectory is assumed to be isotropic curve both in $4 \mathrm{D}$ and in $5 \mathrm{D}: \mathrm{ds}=\mathrm{dS}=0$. From (68)-(70) we obtain solutions

$$
\frac{d y}{d \tau}=0
$$

and

$$
\frac{d y}{d \tau}=-\varepsilon \frac{2 K r}{a_{\varepsilon}^{1 / 2}} .
$$

\section{Astrophysical Applications}

Considering phenomenology of particles motion in $5 \mathrm{D}$ we assume that stationary in 3D space particles, having rest mass, move in spherical or hyperbolic frames in $5 \mathrm{D}$ along geodesics with constant radial coordinate: Eqs. (14), (15) and (27)-(30) or Eqs. (45), (46) and (59)-(62). It is suggested also that in cylindrical frame the matter moves along fifth coordinate in single direction, which is opposite to the antimatter motion.

In case of the space-like fifth dimension the function $f$ from metric (1) is chosen so that its meaning is continuously increasing in intervals 
$I_{n}^{+}=[2 \pi n, \pi+2 \pi n]$ for integer $n$. Since value $r<0$ is inadmissible in cylindrical frame we must assume that function $f$ has discontinuity on the endpoints of $I_{n}^{+}$, which prescribes singularity. It can be avoided if model of binary world consisting of the universe anti-universe pair[26-28] is considered under the assumption that it possesses a large number of copies[29, 30], in which a physical laws are identical. In bulk a space-time half $I_{n}^{-}=[-\pi+2 \pi n, 2 \pi n]$ put into accordance with packet of 4D anti-universes. With condition (5) the intervals $I_{n}^{+}, I_{n}^{-}$contain values of additional coordinate $\chi$. Rotation of one particle with transition to cylindrical coordinates should be interpreted as motion of particle and anti-particle through opposite packets of branes, which conforms to CPT-symmetry of the universe and anti-universe. Thus a birth of the pair particle-antiparticle is assumed to occur in points $y=-+a_{0}, r=0$, after which they move through opposite packets of branes and annihilate, when $y=+-a_{1}, r=0$.

\subsection{Basic Properties of the Pioneer Effect Model}

Recently much attention was attracted to the Pioneer effect, which consists in additional acceleration of spacecrafts Pioneer 10/11[20, 24, 31, 32] $a_{p}=(8.74 \pm 1.33) \times 10^{-8} \mathrm{~cm} \mathrm{~s}^{-2}$ at dictances $15-45$

AU directed to the Sun[33]. We will analyze how much studying models of rotating space conform to this data. Motion of the spacecrafts and the planets will be considered in the frame of the Sun.

For this analysis we must use geodesics of the type I because, as it was shown in Sec. 5, for geodesics with constant radial coordinate they correspond to the neutrally charged particles. Their proper time coincides with coordinate time for trajectories, which are the arcs of circle (16) or hyperbola (47). Also motion of light is assumed to correspond with equation (75), i.e. a light shift along the fifth coordinate is absent.

In the Sun's gravity field motion of the particle with rest mass is described approximately by equations

$$
\frac{d V^{i}}{d S}+\Gamma_{k l}^{i} V^{k} V^{l}=G^{i}
$$

where $G^{i}$ is Schwarzschild-like force vector and left terms correspond to Eqs. (20)-(24) or (53)-(57). Denoting acceleration $W^{i}=\frac{d V^{i}}{d S} \quad$ we divide it into $W^{i}=W_{g}^{i}+W_{F}^{i}$, where vector $W_{F}^{i}$ conforms in case $G^{2}=0, \theta=\pi / 2$ in the neighborhood of point $\left(\tau_{0}, r_{0}, \pi / 2,0,0\right)$ to equations

$$
\begin{gathered}
W_{F}^{0}=G^{0}, \\
W_{F}^{1}=G^{1}+r_{0}\left(\frac{d \varphi}{d S}\right)^{2}, \\
W_{F}^{2}=0, \\
W_{F}^{3}=G^{3}-\frac{2}{r_{0}} \frac{d r}{d S} \frac{d \varphi}{d S}, \\
W_{F}^{4}=G^{4} .
\end{gathered}
$$

The accelerations $W_{g}^{i}$ correspond to Eqs. (31)-(33) or (63)-(65).

By using analogy with motion of particle in central grav ity field in $4 \mathrm{D}$, we take $U_{I}^{1}$ and $d U_{I}^{1} / d S$ for $\chi=\pi / 2$ in spherical coordinates or $\breve{U}_{I}^{1}$ and $d \breve{U}_{I}^{1} / d S$ for $\breve{\chi}=0$ in hyperbolic coordinates as radial velocity and acceleration observed in 4D surface of five-d imensional space-time.

\subsection{Model with Space-Like Fifth Coor dinate}

In spherical coordinates in the neighborhood of point $\left(\tau_{0}, r_{0}, \pi / 2,0, \pi / 2\right)$ Eqs. (31)-(33) correspond to system (6)-(10) reduced to

$$
\begin{gathered}
\frac{d^{2} \tau}{d S^{2}}+\frac{K^{2}}{2} \frac{d \tau}{d S} \frac{d a}{d S}+\frac{K r_{0}^{1 / 2}}{2} \frac{d a}{d S} \frac{d \chi}{d S}=0 \\
\frac{d^{2} a}{d S^{2}}-\frac{K^{2}}{2}\left(\frac{d \tau}{d S}\right)^{2}-\frac{3 r_{0}^{1 / 2}}{2} \frac{d \tau}{d S} \frac{d \chi}{d S}-r_{0}\left(\frac{d \chi}{d S}\right)^{2}=0 \\
\frac{d^{2} \chi}{d S^{2}}+\frac{K\left(3-K^{2} r_{0}\right)}{2 r_{0}^{3 / 2}} \frac{d \tau}{d S} \frac{d a}{d S}+\frac{4-K^{2} r_{0}}{2 r_{0}} \frac{d a}{d S} \frac{d \chi}{d S}=0
\end{gathered}
$$

For close to circular motion (13), (14) non-vanishing five-velocities are written in form

$$
\begin{gathered}
U_{I}^{0}=\sigma+\alpha^{0}, \\
U_{I}^{1}=\alpha^{1}, \\
U_{I}^{4}=-\frac{\sigma K}{a^{1 / 2}}+\alpha^{4},
\end{gathered}
$$

where $\alpha^{i}$ are functions of coordinates. Substitution of $U_{I}^{i}$ in (83)-(85) y ields

$$
\begin{gathered}
\frac{d \alpha^{0}}{d S}=-\frac{K^{2}}{2} \alpha^{0} \alpha^{1}-\frac{K r_{0}^{1 / 2}}{2} \alpha^{1} \alpha^{4} \\
\frac{d \alpha^{1}}{d S}=-\frac{\sigma K^{2}}{2} \alpha^{0}-\frac{\sigma K r_{0}^{1 / 2}}{2} \alpha^{4}+\frac{K^{2}}{2} \alpha^{02}+ \\
+\frac{3 K r_{0}^{1 / 2}}{2} \alpha^{0} \alpha^{4}+r_{0} \alpha^{42} \\
\frac{d \alpha^{4}}{d S}=-K \frac{3-K^{2} r_{0}}{2 r_{0}^{3 / 2}} \alpha^{0} \alpha^{1}-\frac{4-K^{2} r_{0}}{2 r_{0}} \alpha^{1} \alpha^{4}
\end{gathered}
$$


Equation (11) gives

$$
0=2 \sigma \alpha^{0}+\left(1-K^{2} r_{0}\right) \alpha^{02}-2 K r_{0}^{3 / 2} \alpha^{0} \alpha^{4}-\alpha^{12}-r_{0}^{2} \alpha^{42} \text {. }
$$

We consider case $\left|\alpha^{i}\right|<<1$ and assume $\alpha^{4}=0$ on the surface $\chi=\pi / 2$. First and second equations of system (89)-(91) reduce to

$$
\begin{aligned}
& \frac{d \alpha^{0}}{d S}=-\frac{K^{2}}{2} \alpha^{0} \alpha^{1}, \\
& \frac{d \alpha^{1}}{d S}=-\frac{\sigma K^{2}}{2} \alpha^{0},
\end{aligned}
$$

that gives

$$
\sigma \alpha^{0}=\frac{\alpha^{12}}{2}+H,
$$

where $H$ is constant. Substituting this expression into Eq. (94) and choosing $H=0$ we obtain

$$
\frac{d \alpha^{1}}{d S}=-\frac{K^{2} \alpha^{12}}{4} \text {. }
$$

The average spacecraft's velocity on interval 20-50 a.e. is about $\dot{r}_{p}=15 \pm 2 \mathrm{~km} \mathrm{~s}^{-1}$ (See diagram in[24]) and for approximation $S=\tau$ it approximately corresponds to $\alpha^{1}=5 \times 10^{-5}$. Therefore this equation turns out to be

$$
\ddot{r}_{p}=-\frac{K^{2} \dot{r}_{p}^{2}}{4}
$$

that with $\ddot{r}_{p}=-a_{p}$ yields $K=(3.94 \pm 1) \times 10^{-10}$ $\mathrm{cm}^{-1 / 2}$. For this value and made choice of $S, H$, Eq. (95) conforms to (92) without small higher-order terms.

Additional acceleration of Pioneer 11 and its predicted magnitude are contained in Table 1 .

Table 1. Distance from Sun to Pioneer $11 r_{p}$, in AU, its velocity $\dot{r}_{p}$, in $\mathrm{km} \mathrm{s}^{-1}$, unmodeled acceleration $\widetilde{a}_{p}$, in $10^{-8} \mathrm{~cm} \mathrm{~s}^{-2}$ (See plans and figure in[20]) and predicted magnitude of acceleration $\left|\ddot{r}_{p}\right|$, in $10^{-8}$ $\mathrm{cm} \mathrm{s}^{-2}$

\begin{tabular}{|c|c|c|c|}
\hline$r_{p}$ & 6 & 12 & 20 \\
\hline$\dot{r}_{p}$ & $5.7 \pm 0.6$ & $12 \pm 1.5$ & $14 \pm 1.5$ \\
\hline$\widetilde{a}_{p}$ & $0.7 \pm 1.5$ & $6.2 \pm 1.9$ & $9 \pm 0.4$ \\
\hline$\left|\ddot{r}_{p}\right|$ & $1.26 \pm 0.75$ & $5.6 \pm 3.3$ & $7.6 \pm 4.6$ \\
\hline
\end{tabular}

\subsection{Additional Acceleration of Planets}

In this section we will test proposed model by finding additional acceleration for planets of the solar system and comparing them with observations data. Further we will use following denotations: $\gamma$ is gravity constant, $M$ is the Sun's mass, $\Omega$ is its semimajor axis, $e$ is eccentricity, $n=\sqrt{\gamma M / \Omega^{3}}$ is unperturbed Keplerian mean motion, $P=2 \pi / n$ is orbital period and $\xi$ is eccentric anomaly.

Table 2. Semimajor axes $\Omega$ in $\mathrm{AU}$, eccentricities $e$, orbital periods $P$ in years, mean squared radial velocities $\langle\dot{r}\rangle$ in $10^{4} \mathrm{~cm} \mathrm{~s}{ }^{-1}$, coordinate $r$, predicted magnitude of additional radial accelerations $\left|A_{p}\right|$ in $10^{-13} \mathrm{~cm} \mathrm{~s}^{-2}$ and determined from observations anomalous radial accelerations $A_{o b s}$ in $10^{-13} \mathrm{~cm} \mathrm{~s}^{-2}$ for the planets[21] and asteroid Icarus[34]

\begin{tabular}{|c|c|c|c|c|c|c|}
\hline & Yupiter & Saturn & Uranus & Neptune & Pluto & Icarus \\
\hline$\Omega$ & 5.2 & 9.5 & 19.19 & 30.06 & 39.48 & 1.077 \\
\hline$e$ & 0.048 & 0.056 & 0.047 & 0.008 & 0.248 & 0.826 \\
\hline$P$ & 11.86 & 29.45 & 84.07 & 63.72 & 248.02 & 1.12 \\
\hline$\langle\dot{r}\rangle$ & 4.437 & 3.809 & 2.242 & 0.3095 & 8.386 & 190.9 \\
\hline$\left|A_{p}\right|$ & $7.6 \pm 5$ & $5.6 \pm 3.5$ & $2 \pm 1.2$ & $0.037 \pm$ & $27 \pm 16$ & $(14.2 \pm$ \\
\hline$A_{o b s}$ & $100 \pm 700$ & $(-1.34 \pm$ & $(0.058 \pm$ & 0.022 & & $8.4) \times 10^{5}$ \\
\hline
\end{tabular}


Parameters of motion are given by equations

$$
r=\Omega(1-e \cos \xi), n t=\xi-e \sin \xi .
$$

Differentiation of these relations with respect to $t$ yields

$$
\dot{r}=\Omega e \sin \xi \dot{\xi}, \dot{\xi}=\frac{n}{1-e \cos \xi}
$$

and radial velocity is rewritten as

$$
\dot{r}=\frac{n \Omega e \sin \xi}{1-e \cos \xi} .
$$

A mean squared radial velocity during half-period

$$
\langle\dot{r}\rangle=\left(\frac{2}{P} \int_{0}^{P / 2} \dot{r}^{2} d t\right)^{1 / 2}
$$

after fo llowing from (99) substitution

$$
d t=\frac{1}{n}(1-e \cos \xi) d \xi
$$

will be

$$
\langle\dot{r}\rangle=\left(\frac{4 \pi e^{2} \Omega^{2}}{P^{2}} \int_{0}^{\pi} \frac{\sin ^{2} \xi}{1-e \cos \xi} d \xi\right)^{1 / 2} .
$$

Values $\langle\dot{r}\rangle$ for the planets, corresponding them Pioneer-like acceleration

$$
A_{p}=-\frac{K^{2}\langle\dot{r}\rangle^{2}}{4}
$$

and anomalous accelerations of planets $A_{o b s}$, obtained from observations are in Table 2 . Predicted additional radial acceleration for Yupiter, Saturn, Uranus is with in the observation error and for asteroid Icarus it is close to upper limit of $A_{o b s}$.

\subsection{Model with Time-Like Fifth Coordinate}

In hyperbolic coordinates in the neighborhood of point $\left(\breve{\tau}_{0}, \breve{r}_{0}, \pi / 2,0,0\right)$ Eqs. (63)-(65) correspond to system (38)-(42) reduced to

$$
\begin{gathered}
\frac{d^{2} \breve{\tau}}{d S^{2}}-\frac{\breve{K}^{2}}{2} \frac{d \breve{\tau}}{d S} \frac{d \breve{a}}{d S}-\frac{\breve{K}_{0}^{1 / 2}}{2} \frac{d \breve{a}}{d S} \frac{d \breve{\chi}}{d S}=0 \\
\frac{d^{2} \breve{a}}{d S^{2}}+\frac{\breve{K}^{2}}{2}\left(\frac{d \breve{\tau}}{d S}\right)^{2}+\frac{3 \breve{r}_{0}^{1 / 2}}{2} \frac{d \breve{\tau}}{d S} \frac{d \breve{\chi}}{d S}+\breve{r}_{0}\left(\frac{d \breve{\chi}}{d S}\right)^{2}=0 \\
\frac{d^{2} \breve{\chi}}{d S^{2}}+\frac{\breve{K}\left(3+\breve{K}^{2} \breve{r}_{0}\right)}{2 \breve{r}_{0}^{3 / 2}} \frac{d \breve{\tau}}{d S} \frac{d \breve{a}}{d S}+\frac{4+\breve{K}^{2} \breve{r}_{0}}{2 \breve{r}_{0}} \frac{d \breve{a}}{d S} \frac{d \breve{\chi}}{d S}=0
\end{gathered}
$$

For close to hyperbolic motion (45) non-vanishing five-velocities are written in form

$$
\begin{gathered}
\breve{U}_{I}^{0}=\sigma+\breve{\alpha}^{0}, \\
\breve{U}_{I}^{1}=\breve{\alpha}^{1}, \\
\breve{U}_{I}^{4}=-\frac{\sigma \widetilde{K}}{\breve{a}^{1 / 2}}+\breve{\alpha}^{4},
\end{gathered}
$$

where $\breve{\alpha}^{i}$ are functions of coordinates. Substitution of
$\widetilde{U}_{I}^{i}$ in (105)-(107) yields

$$
\begin{gathered}
\frac{d \breve{\alpha}^{0}}{d S}=\frac{\breve{K}^{2}}{2} \breve{\alpha}^{0} \breve{\alpha}^{1}+\frac{\breve{K} \breve{r}_{0}^{1 / 2}}{2} \breve{\alpha}^{1} \breve{\alpha}^{4} \\
\frac{d \breve{\alpha}^{1}}{d S}=\frac{\sigma \breve{K}^{2}}{2} \breve{\alpha}^{0}+\frac{\sigma \breve{K}_{0}^{1 / 2}}{2} \breve{\alpha}^{4}-\frac{\breve{K}^{2}}{2} \breve{\alpha}^{02}-\frac{3 \breve{K}_{0}^{1 / 2}}{2} \breve{\alpha}^{0} \breve{\alpha}^{4}-\breve{r}_{0} \breve{\alpha}^{42} \\
\frac{d \breve{\alpha}^{4}}{d S}=-\breve{K}^{2} \frac{3+\breve{K}^{2} \breve{r}_{0}}{2 \breve{r}_{0}^{3 / 2}} \breve{\alpha}^{0} \breve{\alpha}^{1}-\frac{4+\breve{K}^{2} \breve{r}_{0}}{2 \breve{r}_{0}} \breve{\alpha}^{1} \breve{\alpha}^{4} .
\end{gathered}
$$

Equation (43) gives

$$
0=2 \sigma \breve{\alpha}^{0}+\left(1+\breve{K}^{2} \breve{r}_{0}\right) \breve{\alpha}^{02}+2 \breve{K}^{3 / 2} \breve{\alpha}^{0} \breve{\alpha}^{4}-\breve{\alpha}^{12}+\breve{r}_{0}^{2} \breve{\alpha}^{42} \text {. }
$$

We consider case $\left|\breve{\alpha}^{i}\right|<<1$ and assume $\breve{\alpha}^{4}=0$ on the surface $\breve{\chi}=0$. Equations (111), (112) reduce to

$$
\begin{aligned}
& \frac{d \breve{\alpha}^{0}}{d S}=\frac{\breve{K}^{2}}{2} \breve{\alpha}^{0} \breve{\alpha}^{1}, \\
& \frac{d \breve{\alpha}^{1}}{d S}=\frac{\sigma \breve{K}^{2}}{2} \breve{\alpha}^{0},
\end{aligned}
$$

that gives

$$
\sigma \breve{\alpha}^{0}=\frac{\breve{\alpha}^{12}}{2}+\breve{H}
$$

where $\breve{H}$ is constant. Substituting this expression into Eq. (116) we obtain

$$
\frac{d \breve{\alpha}^{1}}{d S}=\frac{\breve{K}^{2} \breve{\alpha}^{12}}{4}+\frac{\breve{H} \breve{K}^{2}}{2} .
$$

This result doesn't conform to the Pioneer effect, so far as in accordance with this expression with increase of magnitude of radial velocity corresponding growth of acceleration will be positive.

For cylindrical coordinates in case $\breve{V}^{3}=0$ in point $\left(\breve{\tau}_{0}, \breve{r}_{0}, \pi / 2,0,0\right)$ from Eq. (64) we obtain

$$
\frac{d^{2} \breve{r}}{d S^{2}}=-\frac{\breve{K}^{2}}{2}\left(\frac{d \widetilde{\tau}}{d S}\right)^{2} .
$$

With condition $\left|\breve{V}^{1}\right|<<1$, this equation conforms to unmodeled acceleration of Pioneer 10/11 on distance 20-50 a.e., but gives the same acceleration for Pioneer 11 on distance less than 20 a.e. and for planets of the Sun system that contradicts data of observations (Tables 1,2).

\section{Conclusions}

Proposed toy-model of space is based on idea of double manyfold Universe. It is supported by notion that closed geodesic of elementary particle having a rest mass corresponds to motion of the pair particle-antiparticle in the mirror worlds.

Found solutions of geodesics equations corresponding to the particle with a rest mass for cosiderig space-times describe motion in a circle with space-like fifth coordinate and hyperbolic motion with time-like fifth coordinate. Time 
dilation is absence for the solutions corresponded in the Kaluza-Klein model to neutrally charged particle. In this case the angular velocity is inversely proportional to the square root of radius in spherical coordinates. In astrophysical applications the center of the Sun has been chosen as the center of this motion.

Analogy with motion in central gravity field in 4D is employed for determination of velocity and acceleration observed in $4 \mathrm{D}$ sheet for particles moving in $5 \mathrm{D}$ bulk. We obtain approximate solution in the neighborhood of surface with zero fifth coordinate in cylindrical frame for geodesics deviating from having constant radius. With space-like fifth coordinate a body in included 4D space-time with limited velocity will have centripetal acceleration being proportional to square of radial velocity and directed towards center of rotaion of space in $5 \mathrm{D}$. This acceleration is interpreted as action of additional force in $4 \mathrm{D}$. That roughly corresponds to underlying properties of the Pioneer-effect, such as constant additional acceleration of apparatus towards the Sun on distance from 20 to 50 a.e., its increase from 5 to 20 a.e., observed absence of one in motion of planets. However, presence of analogous acceleration in motion of bodies with similar radial velosity, for example, asteroid Icarus, will be significant for confirmation of this model. In case of space-time with time-like fifth coordinate it isn't found geodesics corresponding to this effect.

\section{REFERENCES}

[1] G. Nordstrom, "Uber die Moglichkeit, das Electromagn etishe Feld und das Gravitation Feld zu Vereinigen”, Phyz.Zeitschr., Vol. 15, 504-506, 1914.

[2] T. Kaluza, "Zum Unitatsproblem der Physik", Sitz. Preuss. Akad. Wiss. Phys. Math. K, Vol. 1, pp. 966-972, 1921.

[3] O. Klein, "Quantentheorie und funfdimensionale Relativitatstheorie”, Z. Phys., Vol. 37, pp. 895-906, 1926.

[4] J. M. Overduin and P.S. Wesson, "Kaluza-Klein Gravity", Phys. Rept., Vol. 283, NoNo. 3-4, pp. 303-380, 1997, gr-qc/9805018.

[5] J. Ponce de Leon, "Does force from an extra dimension contradict physics in 4D?” Phys. Lett. B, Vol. 523, NoNo. 3-4, 311-316, 2001, gr-qc/0110063.

[6] A. Herrera-Aguilar and O. V. Kechkin, "Bosonic string Kaluza Klein theory exact solutions using 5D-6D dualities", Mod. Phys. Lett. A, Vol. 16, No. 1, pp. 29-39, 2001, gr-qc/0101007.

[7] J. Ponce de Leon, "Equivalence Between Space-Time-Matter and Brane-World Theories", Mod. Phys. Lett. A, Vol. 16, No. 35, pp. 2291-2303, 2001, gr-qc/0111011.

[8] D.Yu.Tsipenyuk, V.A. Andreev, "5-dimensional extended space model", Presented at 13th General Conference of the European Phy sical Society: Bey ond Einstein - Phy sics of the 21st Century (EPS-13), Bern, Switzerland, 11-15 Jul 2005.

[9] D.Yu.Tsipenyuk, "Field transformation in the extended space model: Prediction and experimental test"., Gravitation and Cosmology, Vol. 7, No. 4(28), pp. 336-338, 2001.

[10] W. B. Belayev, "Cosmological model with movement in fifth dimension”, Space-time and Substance, Vol. 2, No. 2(7), pp. 63-65, 2001, gr-qc/0110099.

[11] K. Koyama, "The cosmological constant and dark energy in braneworlds", Gen. Rel. Grav. Vol. 40, NoNo. 2-3, pp. 421-450, 2008, arXiv:0706.1557.

[12] T. Kobayashi and Y. Takamizu, "Hybrid compactifications and brane gravity in six dimensions", Class. Quant. Grav., Vol.25, No. 1, 015007, 2008, arXiv:0707.0894.

[13] F. Arroja, T. Kobayashi, K. Koyama and T. Shiromizu, "Low energy effective theory on a regularized brane in $6 \mathrm{D}$ gau ged chiral supergravity", JCAP, Vol. 2007, No. 12, 006, 2007, arXiv:0710.2539.

[14] N. Arkani-Hamed, S. Dimopoulos, G. Dvali, "The Hierarchy Problem and New Dimensions at aMillimeter", Phys. Lett. B, Vol.429, NoNo. 3-4, pp. 263-272,1998, hep-ph/9803315.

[15] I. Bars, S.-H. Chen, G. Quelin, "Dual Field Theories In (d-1)+1 Emergent Spacetimes From A Unifying Field Theory In d+2 Spacetime", Phys. Rev. D, Vol. 76, No. 6, 065016, 2007, arXiv:0705.2834.

[16] I. Bars, S.-H. Chen, "Geometry and Symmetry Structures in 2T Gravity”, Phys. Rev. D, Vol. 79, No. 8, 085021, 2009, arXiv:0811.2510.

[17] I. Bars, "Gauge Symmetry in Phase Space, Consequences for Physics and Spacetime", Int. J.Mod. Phys. A, Vol. 25, No. 29, pp. 5235-5252, 2010, arXiv:1004.0688.

[18] K. Trencevski, "Time dependent gravitational potential in the universe and some consequences, Gen. Rel. Grav., Vol. 37, No. 3, pp. 507-519, 2005.

[19] W. B., Belayev, "Cosmological model in 5D, stationarity, yes or no", gr-qc/9903016.

[20] S. G. Turyshev and V. T. Toth, "The Pioneer Anomaly", Living Rev. Rel., Vol. 13, No. 4, 2010, arXiv:1001.3686.

[21] J. D. Anderson, P. A. Laing, E. L. Lau, A. S. Liu, M. M. Nieto and S. G. Turyshev, "Study of anomalous acceleration of Pioneer 10 and 11", Phys. Rev. D, Vol. 65, No. 8, 082004, 2002, gr-qc/0104064.

[22] L. Iorio, "Can the Pioneer anomaly be of gravitational origin? A phenomenological answer", Found. Phys., Vol. 37, No. 6, pp. 897-918, 2007, gr-qc/0610050.

[23] D. Grumiller, "Model for gravity at large distances", Phys. Rev. Lett., Vol. 105, No. 21, 211303, 2010, arXiv:1011.3625.

[24] L. Iorio, "Solar system constraints on a Rindler-type extra-acceleration from modified gravity at large distances", JCAP, Vol. 2011, No. 5, 019, 2011, arXiv:1012.0226.

[25] W .B. Belayev, D.Yu. Tsipenyuk, "Gravi-electromagnetism in five dimensions and moving bodies in galaxy area", Space-time and Substance Vol. 5, No. 2(22), pp. 49-52, 2004, gr-qc/0409056.

[26] A. Linde, "Inflation, Quantum Cosmology and the Anthropic Principle", in "Science and Ultimate Reality: From Quantum to Cosmos", honoring John Wheeler's 90th birthday. J. D. Barrow, P. C. W. Davies, C. L. Harper eds. Cambridge 
University Press, 2003, hep-th/0211048.

[27] S. L. Dubovsky, S. M. Sibiryakov, "Domain walls in noncommutative gauge theories, folded D branes, and communication with mirror world", Nucl. Phys. B, Vol. 691, NoNo. 1-2, pp. 91-110, 2004, hep-th/0401046.

[28] M. Sarrazin, F. Petit, "Equivalence between domain-walls and "noncommutative" two-sheeted spacetimes: Model-independent matter swapping between branes", Phys. Rev. D, Vol. 81, No. 3, 035014, 2010, arXiv:0903.2498.

[29] N. Arkani-Hamed, S. Dimopoulos, G. Dvali, N. Kaloper, "Many fold Universe", JHEP, Vol. 2000, No. 12, 010, 2000, hep-ph/9911386.

[30] G. Dvali, I. Sawicki, A. Vikman, "Dark Matter via Many Copies of the Standard Model", JCAP, Vol. 2009, No. 8, 009, 2009, arXiv:0903.0660.
[31] J. D. Anderson, P. A. Laing, E. L. Lau, A. S. Liu, M. M. Nieto and S. G. Tury shev, "Indication from Pioneer 10/11, Galileo, and Ulysses data, of an apparent anomalous, weak, long-range acceleration", Phys. Rev. Lett., Vol. 81, No. 14, pp. 2858-2861, 1998, gr-qc/9808081.

[32] J. D. Anderson and M.M. Nieto, "Search for a Solution of the Pioneer Anomaly”, Contemp. Phys., Vol. 48, No. 1, pp. 41-54, 2007, arXiv:0709.3866.

[33] S. G. Turyshev, M. M. Nieto, J. D. Anderson, "A Route to Understanding of the Pioneer Anomaly", The XXII Texas Symposium on Relativistic Astrophysics, Stanford U, December 13-17, 2004, edited by P. Chen, E. Bloom, G. Madejski, and V. Petrosian, gr-qc/0503021.

[34] R. H. Sanders, "Solar system constraints on multi-field theories of modified dy namics", Mon. Not. Roy. Astron. Soc., Vol. 370, No. 3, pp. 1519-1528, 2006, astro-ph/0602161. 